\title{
Discretization analysis of bifurcation based nonlinear amplifiers
}

\author{
Sven Feldkord, Marco Reit, and Wolfgang Mathis \\ Institute of Theoretical Electrical Engineering, Leibniz Universität Hannover, Appelstraße 9A, 30167 Hannover, Germany \\ Correspondence to: Marco Reit (reit@tet.uni-hannover.de)
}

Received: 16 January 2017 - Accepted: 5 March 2017 - Published: 21 September 2017

\begin{abstract}
Recently, for modeling biological amplification processes, nonlinear amplifiers based on the supercritical Andronov-Hopf bifurcation have been widely analyzed analytically. For technical realizations, digital systems have become the most relevant systems in signal processing applications. The underlying continuous-time systems are transferred to the discrete-time domain using numerical integration methods. Within this contribution, effects on the qualitative behavior of the Andronov-Hopf bifurcation based systems concerning numerical integration methods are analyzed. It is shown exemplarily that explicit Runge-Kutta methods transform the truncated normalform equation of the Andronov-Hopf bifurcation into the normalform equation of the Neimark-Sacker bifurcation. Dependent on the order of the integration method, higher order terms are added during this transformation.

A rescaled normalform equation of the Neimark-Sacker bifurcation is introduced that allows a parametric design of a discrete-time system which corresponds to the rescaled Andronov-Hopf system. This system approximates the characteristics of the rescaled Hopf-type amplifier for a large range of parameters. The natural frequency and the peak amplitude are preserved for every set of parameters. The Neimark-Sacker bifurcation based systems avoid large computational effort that would be caused by applying higher order integration methods to the continuous-time normalform equations.
\end{abstract}

\section{Introduction}

Early research has shown that the mammalian hearing process is active (Kemp, 1978) and involves an active amplification within the cochlea (Zenner and Gitter, 1987). This amplification characteristic can be modeled by a system near the onset of an Andronov-Hopf bifurcation (Eguíluz et al.,
2000). Regarding this bifurcation based amplifier and after first analog realizations by Stoop et al. $(2005,2007)$ a discrete-time system was developed and implemented on a digital signal processor (DSP) (Reit et al., 2012). This implementation was latterly improved and extended to compare nonlinear amplifiers based on Andronov-Hopf and Neimark-Sacker bifurcations (Feldkord et al., 2016). The Neimark-Sacker bifurcation is also called the AndronovHopf bifurcation for maps (Kuznetsov, 2013).

In this work we analyze the influence of explicit singlestep integration methods on the behavior of systems based on the normalform equation of the Andronov-Hopf bifurcation. This is done exemplarily for the explicit Euler method and extended to explicit Runge-Kutta methods. Lastly, a rescaled normalform equation of the Neimark-Sacker bifurcation is derived that approximates the characteristics of the rescaled Andronov-Hopf bifurcation within the discrete-time domain while preserving the natural frequency and the peak amplitude.

\section{Fundamentals of the Andronov-Hopf and the Neimark-Sacker bifurcation}

The analysis in this work focusses on the truncated normalform equation of the Andronov-Hopf bifurcation (Kuznetsov, 2013)

$$
\frac{\mathrm{d} z}{\mathrm{~d} t}=\left(\mu+\mathrm{i} \omega_{0}\right) z+\sigma|z|^{2} z
$$

where $z \in \mathbb{C}$ is the system variable, $\mu \in \mathbb{R}$ the bifurcation parameter, i the imaginary unit, $\sigma \in \mathbb{C}$ the first nonlinearity coefficient that determines the type of the bifurcation and $\omega_{0} \in \mathbb{R}$ the natural frequency. For $\Re\{\sigma\}<0$, the system is supercritical, branching a stable limit cycle for $\mu>0$ from a stable fixed point $(\mu \leq 0)$ that loses stability. On the contrary for $\Re\{\sigma\}>0$, the system exhibits an unstable limit cycle for 
$\mu<0$ that shrinks and disappears at the point of bifurcation $(\mu=0)$ where the encircled stable fixed point loses stability. Here, for $\mu \geq 0$, the system has an unstable fixed point. A special characteristic of Eq. (1) is the mapping of an additive sinusoidal excitation signal $a_{0} e^{\mathrm{i} \omega t}$ to a sinusoidal output signal $z_{0} e^{\mathrm{i}(\omega t+\phi)}$ without harmonic distortions (Eguíluz et al., 2000; Stoop et al., 2005; Reit et al., 2016).

Similar to the normalform equation of the Andronov-Hopf bifurcation in the continuous-time domain, the truncated normalform equation of the Neimark-Sacker bifurcation in the discrete-time domain (Kuznetsov, 2013),

$z \longmapsto e^{\mathrm{i} \theta} z\left(1+\alpha+d|z|^{2}\right)$

is analyzed. As before, $z$ is the complex system variable. The real-valued bifurcation parameter is denoted by $\alpha$. Similar to $\sigma$ of the Andronov-Hopf system, the type of bifurcation is dependent on the sign of $\Re\{d\}$ while the bifurcation occurs at $\alpha=0$. The Neimark-Sacker bifurcation based system is embedded into a continuous-time environment by iterating the map Eq. (2) with equidistant timesteps $h$. From this iteration, the relation $\theta=h \cdot \omega_{0}$ results. When using AndronovHopf and Neimark-Sacker bifurcation based systems as nonlinear amplifiers, only the supercritical case with $\mu<0$ is considered. Adding an excitation signal for $\mu>0$, the supercritical case results in synchronization problems (Pikovsky et al., 2003). The subcritical case is unstable and therefore neglected. For modeling the cochlea, a $\omega_{0}$-rescaled truncated normalform equation of the Andronov-Hopf bifurcation with an added excitation term $a(t)$,

$\frac{\mathrm{d} z}{\mathrm{~d} t}=\omega_{0}(\mu+\mathrm{i}) z+\omega_{0} \sigma|z|^{2} z+\omega_{0} a(t)$,

was introduced by Stoop et al. (2007). The special property of Eq. (3) is that the amplitude response to a sinusoidal excitation signal $a(t)=a_{0} e^{\mathrm{i} \omega t}$ is independent of the absolute value of the natural frequency. Here, the fraction $\omega / \omega_{0}$ determines the output amplitude for a given set of $\mu, \sigma$ and $a_{0}$. Recently, the rescaled truncated normalform equation of the Andronov-Hopf bifurcation with excitation was implemented on a DSP platform (Reit et al., 2012). In a comparative study of the amplitude responses, the truncated normalform equation of the Neimark-Sacker bifurcation with the excitation $a(n)$,

$z_{n+1}=e^{i \theta} z_{n}\left(1+\alpha+d\left|z_{n}\right|^{2}\right)+a(n)$,

was implemented and analyzed for $a(n)=a_{0} e^{\mathrm{i} \omega h n}$ (Feldkord et al., 2016). It can be shown, that the input-output behavior of the Neimark-Sacker system is very similar to that of the not rescaled Andronov-Hopf system Eq. (1). Thus, to approximate the behavior of the $\omega_{0}$-rescaled Andronov-Hopf system, an appropriate normalform equation of the NeimarkSacker bifurcation is desirable which is introduced later in this work.
Since we examine the purpose of digital processing applications, where real-time processing and low latency are the main goals, only explicit integration methods are considered to implement the Andronov-Hopf bifurcation based systems. The aforementioned DSP-Implementation uses the RungeKutta method of 4 th order.

\section{Analysis of the numerically integrated normalform equation of the Andronov-Hopf bifurcation}

The following section focusses on the qualitative changes of the system behavior due to a discrete-time implementation. We use the rescaled truncated normalform equation of the Andronov-Hopf bifurcation in Eq. (3). The intuitive approach is to transform Eq. (3) into a system in the discretetime domain using a numerical integration method. Then, the resulting difference equation can be analyzed. Due to the nonlinearity of the equation, mixed terms of the state variable and the excitation term can occur. This leads to complicated expressions that makes an analytical approach almost impossible. Therefore, the following analysis focusses on the Andronov-Hopf system in Eq. (3) without the excitation.

To illustrate the transformation of the system parameters due to an integration method, the explicit Euler method is applied and the resulting iterative map

$z \longmapsto z+h \omega_{0}\left((\mu+\mathrm{i}) z+\sigma|z|^{2} z\right)$

is compared to Eq. (2). Equation (5) has the complex conjugated eigenvalues $\lambda_{1,2}=1+h \omega_{0}(\mu \pm i)$. Since the eigenvalues of the Neimark-Sacker normalform equation are known as $e^{ \pm \mathrm{i} \theta}(1+\alpha)$, a comparison leads to

$\alpha=-1+\sqrt{h^{2} \omega_{0}^{2}+\left(h \omega_{0} \mu+1\right)^{2}}$,

$\theta=\arg \left(1+h \omega_{0}(\mu \pm \mathrm{i})\right)$.

Moreover, the nonlinearity coefficient $d$ can be calculated by

$d=e^{-\mathrm{i} \theta} h \omega_{0} \sigma$.

It can be concluded that the application of the explicit Euler method to Eq. (3), omitting the excitation, results in a system that is equivalent to the truncated normalform equation of the Neimark-Sacker bifurcation Eq. (2). An important aspect of this mapping is the addition of a bifurcation offset. Thus, the bifurcation in the discrete-time domain does not occur at $\mu=0$. The mapping also changes the natural frequency as well as the coefficient $d$ of the nonlinear term. The nonlinearity coefficient of the system is scaled by $h \omega_{0}$ and rotated by $\theta$ (cf. Eq. 8). Hence, the type of bifurcation can change from supercritical to subcritical and vice versa when a change in the sign of $\Re\{d\}$ depending on $\theta$ occurs. Regarding the relation Eq. (7), the nonlinearity coefficient is dependent on $\mu$ besides $h \omega_{0}$. The explicit Euler method often requires very small timesteps to satisfactorily approximate the 
solution of very simple differential equations. To overcome this issue, explicit Runge-Kutta methods are preferred. The set of complex-valued terms that are equivalent to the right hand side of the not truncated normalform equation of the Andronov-Hopf bifurcation can be defined as

$$
A=\left\{\sum_{n=0}^{k} \xi_{n}|z|^{2 n} \cdot z \mid \xi_{n} \in \mathbb{C}, k \in \mathbb{N} \backslash\{0\}\right\} .
$$

Every algorithmic step of an explicit Runge-Kutta method (Stoer and Bulirsch, 1978) applied to an Andronov-Hopf system can be generalized to either an insertion $f(g(z))$ or an addition $b \cdot f(z)+c \cdot g(z)$ with $f, g \in A$ and $b, c \in \mathbb{C}$. The addition $b \cdot f(z)+c \cdot g(z)$ is also an element of $A$. The above generalization is only possible under the condition $f(g(z)) \in A$. Assuming $f(g(z)) \notin A$, any further operations relying on $f(g(z))$ would not result in elements of the set $A$.

Dependent on the order of the Runge-Kutta method, the insertion $f(g(z))$ is an operation that can occur multiple times through the calculation of one timestep. It can be shown that one single insertion $f(g(z))$ with $k=K$ for $f(z)$ and $k=M$ for $g(z)$, which calculates the highest exponent, results in an element of $A$ with $k=2 \cdot M+2 \cdot K+2 \cdot M \cdot K$. The resulting terms of any explicit Runge-Kutta method are elements of the set $A$. Thus, they are also terms of the right hand side of the not truncated normalform equation of the Neimark-Sacker bifurcation. It can be concluded that any explicit Runge-Kutta method maps any kind of normalform equation of the Andronov-Hopf bifurcation to a normalform equation of the Neimark-Sacker bifurcation. The value of $k$ for the highest term by using the Runge-Kutta method of 4th order applied to Eq. (3) without excitation can be calculated by the scheme above to $k=40$.

The normalform equation is given completely by the coefficients $\xi_{n}$. The truncated normalform Eq. (2) is obtained by omitting all higher order terms with $n>1$. This can be used for an approximation that is easy to analyze. However, the higher order nonlinearities might change the qualitative behavior of the bifurcation (Reit et al., 2016). Using the system as amplifier, for small values of the excitation amplitude and near the point of bifurcation, the higher order terms cannot be neglected due to large nonlinearity coefficients, e.g. $\Re\left\{\xi_{2}\right\} \approx$ 0.42 for $h=1 / 48 \mathrm{kHz}^{-1}, \omega_{0}=2 \pi \cdot 5 \mathrm{kHz}, \mu=-0.1$ and $\sigma=-1$. Thus, any analysis of the truncated normalform equation must be taken with care and a higher order term approximation should be considered.

\section{Derivation of the Rescaled Neimark-Sacker Bifurcation}

From Sect. 3, we know that explicit Runge-Kutta methods map an Andronov-Hopf system to a NeimarkSacker system. Moreover, the steady-state response of the driven Neimark-Sacker bifurcation resembles the driven Andronov-Hopf system for a wide range of parameters.
In this section, we introduce a rescaled version of the Neimark-Sacker bifurcation based amplifier. To understand the process of reparametrization, some insights into the mapping of the Andronov-Hopf bifurcation to the NeimarkSacker bifurcation are required. One of the conditions for the Andronov-Hopf bifurcation is the crossing of the imaginary axis by a complex conjugated pair of eigenvalues. For the Neimark-Sacker bifurcation, the eigenvalues cross the unit circle. The most simple mapping is done by applying the explicit Euler method to the differential Eq. (1). The iterative map Eq. (5) results, that resembles the normalform equation of the Neimark-Sacker bifurcation of the same degree since the cubic nonlinearity is the highest order term. When the normalform equation of the Andronov-Hopf bifurcation is rescaled and integrated by the explicit Euler method, the terms $h \mu$ and $h \sigma$ are rescaled by $\omega_{0}$,

$z \longmapsto z\left(1+h \omega_{0} \mu+\mathrm{i} h \omega_{0}+h \omega_{0} \sigma|z|^{2}\right)$.

In order to transfer this rescaling to the normalform equation of the Neimark-Sacker bifurcation, it is considered that in the discrete-time domain not the natural frequency itself but $\theta=h \omega_{0}$ is the parameter of interest. Modifying the parameters $\alpha$ and $d$ in Eq. (2) by multiplication with $\theta$, the $\theta$-rescaled truncated normalform equation of the NeimarkSacker bifurcation

$z \longmapsto e^{\mathrm{i} \theta} z\left(1+\theta \alpha+\theta d|z|^{2}\right)$

results. Since $\theta \in \mathbb{R}$ and $\pi>\theta>0$ (Kuznetsov, 2013), the bifurcation point at $\alpha=0$ and the sign of the nonlinearity coefficient remain unchanged. The parametric design of the rescaled Neimark-Sacker bifurcation to approximate the rescaled Andronov-Hopf bifurcation is done by setting $\alpha=\mu, \theta=h \omega_{0}$ and $d=\sigma$. By adding the excitation term $a_{0} e^{\mathrm{i} h \omega n}$, we can compute algebraic amplitude responses for the Neimark Sacker system Eq. (4), the rescaled NeimarkSacker system Eq. (11) and the Runge-Kutta 4 method applied to the rescaled Andronov-Hopf bifurcation (Feldkord et al., 2016). In the excitation term, $n$ is the iteration count and $h \cdot \omega$ the scaled excitation frequency. An algebraic equation for the input-output relation can also be given for the Andronov-Hopf bifurcation in the continuous-time domain (Eguíluz et al., 2000; Reit et al., 2016). Applying the RungeKutta 4 method analytically to the differential equation with excitation, mixed terms of the state variable and the excitation occur. Thus, the differential equation does not map to the normalform equation of the Neimark-Sacker bifurcation. In this case, an algebraic input-output relation is difficult or impossible to compute.

The amplitude responses of the rescaled Neimark-Sacker bifurcation compared to the amplitude response of the rescaled Andronov-Hopf bifurcation, the Neimark-Sacker bifurcation and the difference equation resulting from the Runge-Kutta 4 method applied to the rescaled AndronovHopf bifurcation are shown in Fig. 1a). Besides the inevitable 
(a)
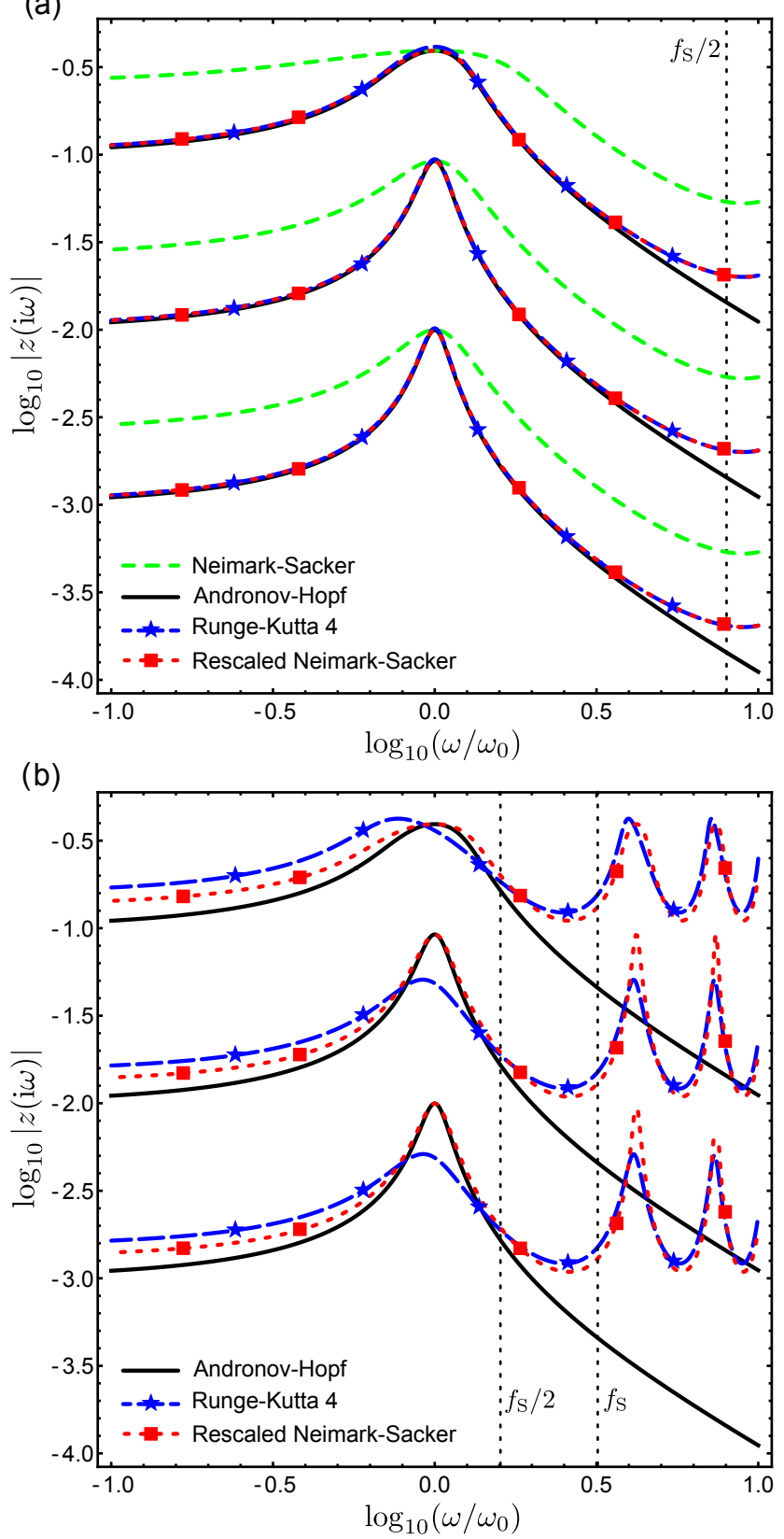

Figure 1. Amplitude responses with $h=1 / f_{\mathrm{S}}=1 / 48 \mathrm{kHz}^{-1}, \sigma=$ $d=-1, \mu=\alpha=-0.1$ and $\omega_{0}=2 \pi \cdot 3 \mathrm{kHz}$ (a) respectively $\omega_{0}=$ $2 \pi \cdot 15 \mathrm{kHz}$ (b). The input amplitudes are $0.1,0.01$ and 0.001 .

periodicity of the discrete-time systems in the frequency, the rescaled Andronov-Hopf and the rescaled Neimark-Sacker systems match very well. On the contrary, the NeimarkSacker system shows a wider bandwidth and is only equal to the Andronov-Hopf system in the peak amplitude and the natural frequency. The rescaled Neimark-Sacker bifurcation shows only small differences in amplitude apart from the natural frequency (Fig. 1b). Using the Runge-Kutta 4 method, the amplitude response deviates for higher natural frequen- cies in amplitude and peak frequency from the solutions of the Andronov-Hopf system (Fig. 1b).

\section{Conclusions}

It is shown that applying an explicit Runge-Kutta method to the truncated normalform of the Andronov-Hopf bifurcation leads to a discrete-time system represented by an iterative map which is equal to the normalform equation of the Neimark-Sacker bifurcation with higher order terms.

For the use as amplifier where the input-output characteristic is approximately independent of the natural frequency, a rescaled truncated normalform equation of the NeimarkSacker bifurcation is introduced. A comparison of the implemented systems is given. It reveals the advantage of designing the Neimark-Sacker bifurcation based system a priori over using an integration method to implement the continuous-time Andronov-Hopf bifurcation based system on a digital platform. It is a much better approximation to the behavior of the rescaled Andronov-Hopf bifurcation. It preserves the peak amplitude and the natural frequency.

Competing interests. The authors declare that they have no conflict of interest.

The publication of this article was funded by the open-access fund of Leibniz Universität Hannover.

Edited by: J. Anders

Reviewed by: B. Schlecker and one anonymous referee

\section{References}

Eguíluz, V. M., Ospeck, M., Choe, Y., Hudspeth, A., and Magnasco, M. O.: Essential Nonlinearities in Hearing, Phys. Rev. Lett., 84, 5232, https://doi.org/10.1103/PhysRevLett.84.5232, 2000.

Feldkord, S., Reit, M., and Mathis, W.: Implementation of a digital evaluation platform to analyze bifurcation based nonlinear amplifiers, Adv. Radio Sci., 14, 47-50, https://doi.org/10.5194/ars14-47-2016, 2016.

Kemp, D. T.: Stimulated Acoustic Emissions from Within the Human Auditory System, J. Acoust. Soc. Am., 64, 1386-1391, https://doi.org/0.1121/1.382104, 1978.

Kuznetsov, Y. A.: Elements of Applied Bifurcation Theory, in: Applied Mathematical sciences, Vol. 112, edited by: Antman, S. S., Marsden, J. E., and Sirovich, L., Springer, New York, 2004

Pikovsky, A., Rosenblum, M., and Kurths, J.: Synchronization: a universal concept in nonlinear sciences, in: Cambridge Nonlinear Science Series (Book 12), Cambridge University Press, 2003.

Reit, M., Mathis, W., and Stoop, R.: Time-Discrete Nonlinear Cochlea Model Implemented on DSP for Auditory Studies, in: Nonlinear Dynamics of Electronic Systems, Proceedings of NDES 2012, 11-13 July 2012, Wolfenbüttel, Germany, 1-4, 2012 . 
Reit, M., Berens, M., and Mathis, W.: Ambiguities in inputoutput behavior of driven nonlinear systems close to bifurcation, Archives of Electrical Engineering, 65, 337-347, 2016.

Stoer, J. and Bulirsch, R.: Einführung in die Numerische Mathematik II, https://doi.org/10.1007/978-3-662-06866-3, Springer, Berlin, 1978.

Stoop, R., Kern, A., and van der Vyver, J. J.: How Ears Go Electronic, in: 2005 International Symposium on Nonlinear Theory and its Applications (NOLTA2005), 18-21 October 2005, Bruges, Belgium, 533-536, 2005.
Stoop, R., Jasa, T., Uwate, Y., and Martignoli, S.: From Hearing to Listening: Design and Properties of an Actively Tunable Electronic Hearing Sensor, Sensors, 7, 3287-3298, https://doi.org/10.3390/s7123287, 2007.

Zenner, H.-P. and Gitter, A. H.: Die Schallverarbeitung des Ohres - ein Bericht über aufregende Experimente mit mikrochirurgisch isolierten Haarzellen, Physik in unserer Zeit, 18, 97-105, 1987. 